\title{
Synthesis and evaluation of some bioactive compounds having oxygen and nitrogen heteroatom
}

\author{
POONAM YADAV ${ }^{\mathrm{a}}$ and NALINI V PUROHIT ${ }^{\mathrm{b}, *}$ \\ ${ }^{a}$ School of Science and Education, Navrachana University, Vadodara 391 410, India \\ ${ }^{b}$ Department of Chemistry, Faculty of Science, The Maharaja Sayajirao University of Baroda, \\ Vadodara 390 002, India \\ e-mail: nalinipurohit22@gmail.com
}

MS received 8 February 2012; revised 11 April 2012; accepted 26 April 2012

\begin{abstract}
Some new 3,4-disubstituted isocoumarins were synthesized having bioactive pyrazole molecule at 3rd position of isocoumarin moiety $(\mathbf{5 a}, \mathbf{b})$, from isocoumarin -3- carboxylic acid hydrazide $(\mathbf{4} \mathbf{a}, \mathbf{b})$ followed by cyclization with acetyl acetone. A series of isocoumarin derivative having Schiff base as lateral side chain at 3rd position of isocoumarin moiety were also synthesized $(\mathbf{7 a}, \mathbf{b})$, by condensing isocoumarin acid hydrazide and benzaldehyde derivative followed by dehydration. The chemical structures of all the compounds were determined by analytical and spectral method. The lead compounds were screened for antimicrobial and analgesic activities.
\end{abstract}

Keywords. Pyrazole isocoumarins; Schiff base isocoumarins; antibacterial activity; antifungal activity; analgesic activity.

\section{Introduction}

Chemical modification of bioactive components of naturally occurring metabolites is one of the most common approaches in drug discovery for new drugs and improved therapeutic properties. Therefore, in continuation of our research programme aimed at obtaining new beneficial agents, some new isocoumarin derivatives were synthesized containing different nitrogen heterocyclic moities. Careful literature surveys ${ }^{1}$ revealed that pyrazole ring system have occupied a unique position in the design and synthesis of novel biological active agent with remarkable analgesic activity. Inspired by these, the nitrogen heterocyclic moities which were chosen for this series to be introduced in isocoumarin moitites are pyrazole and Schiff base functionalities.

There are two basic approaches to develop a new drug: (i) synthesis of analogues, modifications or derivatives of existing compounds for shortening and improving treatment and (ii) searching for novel structures, that the bacteria has never been presented before. ${ }^{2}$ To pursue this goal, our research efforts are directed to synthesize new pharmacophores.

Pyrazole derivatives are well-established in the literatures as important biologically effective heterocyclic compounds. These derivatives are the subject of many

*For correspondence research studies due to their widespread potential pharmacological activities such as antiinflammatory, ${ }^{3}$ antipyretic, ${ }^{4}$ antimicrobial, ${ }^{5}$ anticancer, ${ }^{6}$ antiviral, ${ }^{7}$ antioxidant, ${ }^{8}$ and anticonvulsant ${ }^{9}$ agents.

Pyrazole-tethered phosphine ligands are useful catalysts for Stille-Kumada cross coupling reactions which has wide applications in natural product synthesis, carbohydrate chemistry and biological research where as Hiyama cross-coupling reactions are important and selective method for producing carbon-carbon bonds. ${ }^{10}$

Schiff bases are widely used for industrial purposes ${ }^{11}$ and also exhibit a broad range of biological activities and hence form an important class of organic compound attracting the attention of organic chemists.

More-over, Schiff bases have found application in drug development for the treatment of hypertension, HIV infection and have been shown to exhibit a broad range of biological activities, including antifungal, antibacterial, antimalarial, antiproliferative, antiinflammatory, antiviral, and antipyretic properties. ${ }^{12}$

Imine or azomethine groups are present in various natural, natural-derived and synthetic compounds. The imine group present in such compounds has been shown to be critical for their biological activities. ${ }^{13}$

Prompted by the observations and the chemotherapeutic value of the nitrogenous chemical scaffolds, and in continuation of our interest in the synthesis of some novel isocoumarin derivatives with different nitrogen heterocycles that may be valuable in designing 
new selective antimicrobial agent, we report here the synthesis of such compounds where efforts have now been made to develop new and safe synthetic bioactive agents and screened them for their antimicrobial activity against Staphylococcus aureus, Escherichia coli, Thielaviopsis paradoxa, Phomopsis mangiferae, Fusarium pallidoroseum, Colletotrichum capsici and analgesic activity in vivo on mice.

\section{Experimental}

\subsection{Materials and methods}

The reagents and the solvents used in this study were of analytical grade and were used without further purification. Melting points were determined in open capillaries and are uncorrected. The purity of the compounds was checked by TLC on silica gel GF254 plates using UV/Iodine as visualizing agent and Merk's silica gel (60-120 mesh) was used for column chromatographic purification. Infrared spectra were recorded on FTIR Perkin Elmer spectrophotometer using potassium bromide optics. ${ }^{1} \mathrm{H}$ NMR spectra were recorded on a Bruker spectrometer (400 MHz) using TMS as internal standard and chemical shifts are given in ppm. Mass spectra were obtained using Thermo Scientific Corporation, DSQ II Mass Spectrometer. Elemental analyses were carried out on Perkin-Elmer C, H, N, S analyzer (Model-2400).

\subsection{General procedure for synthesis of 4-alkyl - isocoumarin -3- carboxylic acid (3a-b)}

$o$-Acetyl benzoic acid $1(2.0 \mathrm{~g}, 0.012 \mathrm{~mol})$, bromo diethyl malonate $2(2.0 \mathrm{ml} 0.012 \mathrm{~mol})$ and anhy. $\mathrm{K}_{2} \mathrm{CO}_{3}$, $(3.53 \mathrm{~g}, 0.022 \mathrm{~mol})$ was refluxed for $10-12 \mathrm{~h}$ in ethyl methyl ketone. Solvent was then removed, water was added and extracted with ethyl acetate. Solvent layer was first washed with sod. bicarbonate and then with water and dried over anhy. $\mathrm{Na}_{2} \mathrm{SO}_{4}$. After removal of solvent, the crude product was purified by column chromatography using petroleum ether $\left(60-80^{\circ} \mathrm{C}\right)$-ethyl acetate to yield intermediate which was then refluxed with conc. $\mathrm{HCl}(9.0 \mathrm{ml})$ and glacial acetic acid $(12.0 \mathrm{ml})$ for $6 \mathrm{~h}$. After that the reaction mixture was cooled and poured in crushed ice and left overnight. The solid product obtained was filtered and recrystallised with methanol.

2.2a 4-Methyl-isocoumarin-3-carboxylic acid ${ }^{14}$ (3a): This compound was obtained as brown crystals, mp: $242^{\circ} \mathrm{C}$; $63.17 \%$ yield; Anal. Calcd $\mathrm{C}_{11} \mathrm{H}_{8} \mathrm{O}_{4}$ (204.0 g):
C, 64.70; H, 3.92; Found: C, 64.58; H, 4.26; ${ }^{1} \mathrm{H}$ NMR $\delta 2.3\left(\mathrm{~s}, 3 \mathrm{H}, \mathrm{CH}_{3}\right), 7.7-8.0$ (m, $3 \mathrm{H}$, aromatic protons), 8.39 (d, 1H, $\left.\mathrm{C}_{8}-\mathrm{H}\right) 11.0(\mathrm{~s}, 1 \mathrm{H}, \mathrm{OH})$; ms: m/z: 204 $\left(\mathrm{M}^{+}\right), 203,189,146,118,77$.

2.2b 4-Ethyl-isocoumarin-3-carboxylic acid ${ }^{14}$ (3b): This compound was obtained as brown crystals, mp: $182^{\circ} \mathrm{C}$; $56.30 \%$ yield; Anal. Calcd $\mathrm{C}_{12} \mathrm{H}_{10} \mathrm{O}_{4}(218.0$ g): C, 66.05; H, 4.58; Found: C, 66.38; H, 4.86; ${ }^{1} \mathrm{H}$ NMR $\delta 1.1\left(\mathrm{t}, 3 \mathrm{H}, \mathrm{CH}_{3}\right), 1.7\left(\mathrm{q}, 2 \mathrm{H}, \mathrm{CH}_{2}\right), 7.7-7.9(\mathrm{~m}, 3 \mathrm{H}$, aromatic protons), 8.4-8.45 (dd, $\left.1 \mathrm{H}, \mathrm{C}_{8}-\mathrm{H}\right) 11.2(\mathrm{~s}, 1 \mathrm{H}$, $\mathrm{OH})$; ms: m/z: $218\left(\mathrm{M}^{+}\right), 217,190,174$ and 146.

\subsection{General procedure for synthesis of 4-alkyl -} isocoumarin - 3- carboxylic acid hydrazide (4a-b)

To a solution of $80 \%$ aq. hydrazine hydrate $(0.3 \mathrm{ml})$, isocoumarin-3-carboxylic acid $3(0.1 \mathrm{~g}, 0.00049 \mathrm{~mol})$ was added portion-wise and after addition was complete, it was refluxed for $15 \mathrm{~min}$. After that, absolute alcohol was added to the reaction mixture which was just enough to get a clear solution and reaction mixture was refluxed for $5 \mathrm{~h}$. After completion of reaction, solvent was distilled off and solid product obtained was recrystallized from ethanol.

2.3a 4-Methyl isocoumarin-3-carboxylic acid hydrazide (4a): This compound was obtained as yellow crystals, mp: $242^{\circ} \mathrm{C} ; 65.00 \%$ yield; Anal. Calcd $\mathrm{C}_{11} \mathrm{H}_{10} \mathrm{~N}_{2} \mathrm{O}_{3}$ (218.0 g): C, 60.55; H, 4.58; N, 12.84; Found: C, 60.14; $\mathrm{H}, 4.73 ; \mathrm{N}, 12.96 ;{ }^{1} \mathrm{H}$ NMR $\delta 2.2\left(\mathrm{~s}, 3 \mathrm{H}, \mathrm{CH}_{3}\right), 6.9(\mathrm{~d}$, $\left.2 \mathrm{H}, \mathrm{NH}_{2}\right), 7.9(\mathrm{t}, 1 \mathrm{H}, \mathrm{NH}), 7.6-7.8(\mathrm{~m}, 3 \mathrm{H}$, aromatic protons), $8.4\left(\mathrm{~d}, 1 \mathrm{H}, \mathrm{C}_{8}-\mathrm{H}\right)$; ms: m/z: $218\left(\mathrm{M}^{+}\right), 202$, 187, 173, 159, 146, 77 and 59.

$2.3 \mathrm{~b}$ 4-Ethyl isocoumarin-3-carboxylic acid hydrazide (4b): This compound was obtained as yellow crystals, mp: $182^{\circ} \mathrm{C}$; $48.71 \%$ yield; Anal. Calcd $\mathrm{C}_{12} \mathrm{H}_{12} \mathrm{~N}_{2} \mathrm{O}_{3}$ (232.0 g): C, 62.06; H, 5.17; N, 12.06; Found: C, 62.31; $\mathrm{H}, 5.42 ; \mathrm{N}, 12.46 ;{ }^{1} \mathrm{H}$ NMR $\delta 1.1\left(\mathrm{t}, 3 \mathrm{H}, \mathrm{CH}_{3}\right), 1.8$ (q, $\left.2 \mathrm{H}, \mathrm{CH}_{2}\right), 7.0\left(\mathrm{~d}, 2 \mathrm{H}, \mathrm{NH}_{2}\right), 7.9(\mathrm{t}, 1 \mathrm{H}, \mathrm{NH}), 7.6-7.8$ (m, $3 \mathrm{H}$, aromatic protons), $8.45\left(\mathrm{dd}, 1 \mathrm{H}, \mathrm{C}_{8}-\mathrm{H}\right)$; ms: $\mathrm{m} / \mathrm{z}: 232\left(\mathrm{M}^{+}\right), 216,201,173,172,146$ and 59.

\subsection{General procedure for synthesis of 3-(3', 5'-dimethyl pyrazole-1-carbonyl)-4-alkyl isocoumarin (5a-b)}

A mixture of isocoumarin carboxylic acid hydrazide $4(0.106 \mathrm{~g}, 0.00048 \mathrm{~mol})$, acetylacetone $(0.05 \mathrm{ml}$, $0.00048 \mathrm{~mol}$ ) and $2 \mathrm{M} \mathrm{HCl}$ was refluxed in methanol 
for $15 \mathrm{~h}$. Solvent was then distilled off and the residue obtained was recrystallised from ethanol.

2.4a 3-(3', 5'-Dimethyl pyrazole-1-carbonyl)-4-methyl isocoumarin (5a): This compound was obtained as white crystals, mp: $220^{\circ} \mathrm{C}$; $72.69 \%$ yield; Anal. Calcd $\mathrm{C}_{16} \mathrm{H}_{14} \mathrm{~N}_{2} \mathrm{O}_{3}$ (282.0 g): C, 68.08; H, 4.96; N, 9.92; Found: C, 68.21; H, 4.53; N, 10.21; ${ }^{1} \mathrm{H}$ NMR $\delta 2.3$ (s, $\left.3 \mathrm{H}, \mathrm{CH}_{3}\right), 2.9\left(\mathrm{~s}, 6 \mathrm{H}, \mathrm{CH}_{3}\right), 7.0(\mathrm{~s}, 1 \mathrm{H},=\mathrm{CH}), 7.5-7.8$ (m, $3 \mathrm{H}$, aromatic protons), $8.3\left(\mathrm{~d}, 1 \mathrm{H}, \mathrm{C}_{8}-\mathrm{H}\right) ; \mathrm{ms}: \mathrm{m} / \mathrm{z}$ $282\left(\mathrm{M}^{+}\right), 267,237,209,173$ and 146.

2.4b 3-(3', 5'-Dimethyl pyrazole-1-carbonyl)-4-ethyl isocoumarin (5b): This compound was obtained as white crystals, mp: $230^{\circ} \mathrm{C}$; $73.45 \%$ yield; Anal. Calcd $\mathrm{C}_{17} \mathrm{H}_{16} \mathrm{~N}_{2} \mathrm{O}_{3}$ (296.0 g): C, 68.91; H, 5.40; N, 9.45; Found: $\mathrm{C}, 68.61 ; \mathrm{H}, 5.73 ; \mathrm{N}, 9.81 ;{ }^{1} \mathrm{H} \mathrm{NMR} \delta 1.3(\mathrm{t}$, $\left.3 \mathrm{H}, \mathrm{CH}_{3}\right), 2.7\left(\mathrm{q}, 2 \mathrm{H}, \mathrm{CH}_{2}\right), 2.9\left(\mathrm{~s}, 6 \mathrm{H}, \mathrm{CH}_{3}\right), 6.2$ (s, $1 \mathrm{H},=\mathrm{CH}), 7.5-7.8(\mathrm{~m}, 3 \mathrm{H}$, aromatic protons $), 8.3(\mathrm{~d}$, $\left.1 \mathrm{H}, \mathrm{C}_{8}-\mathrm{H}\right)$; ms: m/z $295\left(\mathrm{M}^{+}-1\right), 267,254,209$ and 146.

\subsection{General procedure for synthesis of Schiff bases $(7 \mathbf{a}-\mathbf{l})$}

A mixture of isocoumarin-3-carboxylic acid hydrazide $4(0.106 \mathrm{~g}, 0.00048 \mathrm{~mol}), \quad p$-nitro benzaldehyde 6 $(0.0734 \mathrm{~g}, 0.00048 \mathrm{~mol})$ and few drops of conc. $\mathrm{H}_{2} \mathrm{SO}_{4}$ was refluxed in ethanol for $4 \mathrm{~h}$. After the reaction was over, reaction mass was poured into ice, product filtered and recrystallized from ethanol to give white crystals of $7^{\mathrm{a}}$.

2.5a 4-Methyl-isocoumarin-3-carboxylic acid (4'-nitro benzylidene)-hydrazide (7a): This compound was obtained as yellow crystals, mp: $140^{\circ} \mathrm{C} ; 76.28 \%$ yield; Anal. Calcd $\mathrm{C}_{18} \mathrm{H}_{13} \mathrm{~N}_{3} \mathrm{O}_{5}$ (351.0 g): C, 61.53; H, 3.70; N, 11.96; Found: C, 61.82; H, 3.84; N, 12.08; ${ }^{1} \mathrm{H}$ NMR $\delta 2.1\left(\mathrm{~s}, 3 \mathrm{H}, \mathrm{CH}_{3}\right), 7.4(\mathrm{~s}, 1 \mathrm{H},=\mathrm{CH}), 7.5-8.3(\mathrm{~m}, 7 \mathrm{H}$, aromatic protons), $8.4\left(\mathrm{~d}, 1 \mathrm{H}, \mathrm{C}_{8}-\mathrm{H}\right), 9.0(\mathrm{~s}, 1 \mathrm{H}, \mathrm{NH})$; ms: $\mathrm{m} / \mathrm{z}: 353\left(\mathrm{M}^{+}+2\right), 229,213,171,135,71$ and 57.

$2.5 \mathrm{~b}$ 4-Methyl-isocoumarin-3-carboxylic acid (4'-hydroxy benzylidene)-hydrazide (7b): This compound was obtained as white crystals, mp: $242^{\circ} \mathrm{C} ; 74.53 \%$ yield; Anal. Calcd $\mathrm{C}_{18} \mathrm{H}_{14} \mathrm{~N}_{2} \mathrm{O}_{4}$ (322.0 g): C, 67.08; H, 4.34; N, 8.69; Found: C, 67.15; H, 4.30; N, 8.81; ${ }^{1} \mathrm{H}$ NMR $\delta 2.0\left(\mathrm{~s}, 3 \mathrm{H}, \mathrm{CH}_{3}\right), 3.9(\mathrm{~s}, 1 \mathrm{H}, \mathrm{OH}), 7.3(\mathrm{~s}, 1 \mathrm{H},=\mathrm{CH})$, 6.95-8.25 (m, 7H, aromatic protons), $8.35\left(\mathrm{~d}, 1 \mathrm{H}, \mathrm{C}_{8^{-}}\right.$ H), 10.1(s, 1H, NH); ms: m/z: $321\left(\mathrm{M}^{+}-1\right), 307,294$, 290, 213, 122, 71 and 57. 2.5c 4-Methyl-isocoumarin-3-carboxylic acid (4'-methoxy benzylidene)-hydrazide (7c): This compound was obtained as yellow crystals, mp: $160^{\circ} \mathrm{C} ; 83.47 \%$ yield; Anal. Calcd $\mathrm{C}_{19} \mathrm{H}_{16} \mathrm{~N}_{2} \mathrm{O}_{4}$ (336.0 g): C, 67.85; H, 4.76; N, 8.33; Found: C, 67.53; H, 4.77; N, 8.39; ${ }^{1} \mathrm{H}$ NMR $\delta$ $2.0\left(\mathrm{~s}, 3 \mathrm{H}, \mathrm{CH}_{3}\right), 4.0\left(\mathrm{~s}, 3 \mathrm{H}, \mathrm{OCH}_{3}\right), 7.3(\mathrm{~s}, 1 \mathrm{H},-\mathrm{C}=\mathrm{H})$, 7.1-8.3 (m, 7H, aromatic protons), $8.4\left(\mathrm{dd}, 1 \mathrm{H}, \mathrm{C}_{8}-\mathrm{H}\right)$, 9.3 (s, $1 \mathrm{H}, \mathrm{NH}) ; \mathrm{ms}: \mathrm{m} / \mathrm{z}: 336\left(\mathrm{M}^{+}\right), 264,215,187,135$, 71 and 57.

2.5d 4-Methyl-isocoumarin-3-carboxylic acid benzylidenehydrazide (7d): This compound was obtained as white crystals, mp: $230^{\circ} \mathrm{C}$; $83.00 \%$ yield; Anal. Calcd $\mathrm{C}_{18} \mathrm{H}_{14} \mathrm{~N}_{2} \mathrm{O}_{3}$ (306.0 g): C, 70.58; H, 4.57; N, 9.15; Found: C, 70.20; H, 4.90; N, 9.11; ${ }^{1} \mathrm{H}$ NMR $\delta 1.8$ (s, $\left.3 \mathrm{H}, \mathrm{CH}_{3}\right), 6.8(\mathrm{~s}, 1 \mathrm{H},-\mathrm{C}=\mathrm{H}), 7.3-7.7(\mathrm{~m}, 8 \mathrm{H}$, aromatic protons), 8.36-8.4 (d, 1H, $\left.\mathrm{C}_{8}-\mathrm{H}\right), 9.5$ (s, $\left.1 \mathrm{H}, \mathrm{NH}\right)$; ms: m/z: $306\left(\mathrm{M}^{+}\right), 215,202,187,172,147$ and 145.

2.5e 4-Methyl-isocoumarin-3-carboxylic acid (2'-hydroxy benzylidene)-hydrazide (7e): This compound was obtained as white crystals, mp: $208^{\circ} \mathrm{C} ; 59.71 \%$ yield; Anal. Calcd $\mathrm{C}_{18} \mathrm{H}_{14} \mathrm{~N}_{2} \mathrm{O}_{4}$ (322.0 g): C, 67.08; H, 4.34; N, 8.69; Found: C, 67.00; H, 4.46; N, 8.75; ${ }^{1} \mathrm{H}$ NMR $\delta 2.0\left(\mathrm{~s}, 3 \mathrm{H}, \mathrm{CH}_{3}\right), 5.4(\mathrm{~s}, 1 \mathrm{H}, \mathrm{OH}), 7.0-7.8(\mathrm{~m}, 7 \mathrm{H}$, aromatic protons), $8.2(\mathrm{~s}, 1 \mathrm{H},=\mathrm{CH}), 8.3\left(\mathrm{~d}, 1 \mathrm{H}, \mathrm{C}_{8}-\mathrm{H}\right)$, 8.8 (s, 1H, NH); ms: m/z: $322\left(\mathrm{M}^{+}\right), 216,147,121,93$ and 77.

$2.5 f$ 4-Methyl-isocoumarin-3-carboxylic acid (3'-phenyl allylidene)-hydrazide (7f): This compound was obtained as yellow crystals, mp: $180^{\circ} \mathrm{C} ; 50.95 \%$ yield; Anal. Calcd $\mathrm{C}_{20} \mathrm{H}_{16} \mathrm{~N}_{2} \mathrm{O}_{3}$ (332.0 g): C, 72.28; H, 4.81; N, 8.43; Found: C, 72.26; H, 5.03; N, 8.60; ${ }^{1} \mathrm{H}$ NMR $\delta$ $2.1\left(\mathrm{~s}, 3 \mathrm{H}, \mathrm{CH}_{3}\right), 5.8\left(\mathrm{~s}, 1 \mathrm{H},=\mathrm{C}_{2^{\prime}} \mathrm{H}\right), 6.5\left(\mathrm{~s}, 1 \mathrm{H},=\mathrm{C}_{3^{\prime}} \mathrm{H}\right)$, $7.5\left(\mathrm{~s}, 1 \mathrm{H},=\mathrm{C}_{1^{\prime}} \mathrm{H}\right), 7.0-7.6(\mathrm{~m}, 8 \mathrm{H}$, aromatic protons), $8.2(\mathrm{~s}, 1 \mathrm{H}, \mathrm{NH}), 8.3$ (d, 1H, $\left.\mathrm{C}_{8}-\mathrm{H}\right) ; \mathrm{ms}: \mathrm{m} / \mathrm{z}: 332\left(\mathrm{M}^{+}\right)$, 317, 240, 228, 214, 130, 116 and 103.

$2.5 \mathrm{~g}$ 4-Ethyl-isocoumarin-3-carboxylic acid (4'-nitro benzylidene)-hydrazide (7g): This compound was obtained as yellow crystals, mp: $230^{\circ} \mathrm{C} ; 70.93 \%$ yield; Anal. Calcd $\mathrm{C}_{19} \mathrm{H}_{15} \mathrm{~N}_{3} \mathrm{O}_{5}$ (365.0 g): C, 62.46; H, 4.10; N, 11.50; Found: C, 62.51; H, 4.00; N, $11.72 ;{ }^{1} \mathrm{H}$ NMR $\delta 1.4\left(\mathrm{t}, 3 \mathrm{H}, \mathrm{CH}_{3}\right), 2.5\left(\mathrm{q}, 2 \mathrm{H}, \mathrm{CH}_{2}\right), 7.3(\mathrm{~s}, 1 \mathrm{H},=\mathrm{CH})$, 7.5-8.3 (m, 7H, aromatic protons), $8.4\left(\mathrm{~d}, 1 \mathrm{H}, \mathrm{C}_{8}-\mathrm{H}\right)$, 8.8 (s, 1H, NH); ms: m/z: $366\left(\mathrm{M}^{+}+1\right), 319,305,242$, $187,122,71$ and 57.

2.5h 4-Ethyl-isocoumarin-3-carboxylic acid (4'-hydroxy benzylidene)-hydrazide (7h): This compound was 
obtained as white crystals, mp: $110^{\circ} \mathrm{C} ; 74.00 \%$ yield; Anal. Calcd $\mathrm{C}_{19} \mathrm{H}_{16} \mathrm{~N}_{2} \mathrm{O}_{4}$ (336.0 g): C, 67.85; H, 4.76; $\mathrm{N}, 8.33$; Found: C, 67.60; H, 4.93; N, 8.65; ${ }^{1} \mathrm{H}$ NMR $\delta$ $1.1\left(\mathrm{t}, 3 \mathrm{H}, \mathrm{CH}_{3}\right), 2.5$ (q, 2H, $\left.\mathrm{CH}_{2}\right), 4.9(\mathrm{~s}, 1 \mathrm{H}, \mathrm{OH}), 7.3$ $(\mathrm{s}, 1 \mathrm{H},=\mathrm{CH}), 7.1-8.3(\mathrm{~m}, 7 \mathrm{H}$, aromatic protons), 8.45 (d, $\left.1 \mathrm{H}, \mathrm{C}_{8}-\mathrm{H}\right), 10.1(\mathrm{~s}, 1 \mathrm{H}, \mathrm{NH}) ; \mathrm{ms}: \mathrm{m} / \mathrm{z}: 336\left(\mathrm{M}^{+}\right)$, 308, 278, 213, 165 and 122.

2.5i 4-Ethyl-isocoumarin-3-carboxylic acid (4'-methoxy benzylidene)-hydrazide (7i): This compound was obtained as yellow crystals, mp: $170^{\circ} \mathrm{C} ; 83.69 \%$ yield; Anal. Calcd $\mathrm{C}_{20} \mathrm{H}_{18} \mathrm{~N}_{2} \mathrm{O}_{4}$ (350.0 g): C, 68.57; H, 5.14; N, 8.00; Found: C, 68.70; H, 5.44; N, 8.26; ${ }^{1} \mathrm{H}$ NMR $\delta$ $1.0\left(\mathrm{t}, 3 \mathrm{H}, \mathrm{CH}_{3}\right), 2.0\left(\mathrm{q}, 2 \mathrm{H}, \mathrm{CH}_{2}\right), 3.5\left(\mathrm{~s}, 3 \mathrm{H}, \mathrm{OCH}_{3}\right)$, $6.9(\mathrm{~s}, 1 \mathrm{H},-\mathrm{C}=\mathrm{H}), 7.1-7.6(\mathrm{~m}, 7 \mathrm{H}$, aromatic protons), $8.4\left(\mathrm{~d}, 1 \mathrm{H}, \mathrm{C}_{8}-\mathrm{H}\right), 10.3$ (s, $\left.1 \mathrm{H}, \mathrm{NH}\right)$; ms: m/z: $350\left(\mathrm{M}^{+}\right)$, 262, 202, 199, 187, 185, 160 and 146.

2.5j 4-Ethyl-isocoumarin-3-carboxylic acid benzylidenehydrazide (7j): This compound was obtained as white crystals, mp: $170^{\circ} \mathrm{C} ; 76.94 \%$ yield; Anal. Calcd $\mathrm{C}_{19} \mathrm{H}_{16} \mathrm{~N}_{2} \mathrm{O}_{3}$ (320.0 g): C, 71.25; H, 5.00; N, 8.75; Found: C, 71.33; H, 5.34; N, 9.04; ${ }^{1} \mathrm{H}$ NMR $\delta 1.1(\mathrm{t}$, $\left.3 \mathrm{H}, \mathrm{CH}_{3}\right), 2.0\left(\mathrm{q}, 2 \mathrm{H}, \mathrm{CH}_{2}\right), 6.8(\mathrm{~s}, 1 \mathrm{H},-\mathrm{C}=\mathrm{H}), 7.3-7.7$ (m, $8 \mathrm{H}$, aromatic protons), 8.36-8.4 $\left(\mathrm{d}, 1 \mathrm{H}, \mathrm{C}_{8}-\mathrm{H}\right), 9.0$ (s, 1H, NH); ms: m/z: $320\left(\mathrm{M}^{+}\right), 305,202,173,146,77$ and 57.

$2.5 \mathrm{k}$ 4-Ethyl-isocoumarin-3-carboxylic acid (2'-hydroxy benzylidene)-hydrazide (7k): This compound was obtained as white crystals, mp: $220^{\circ} \mathrm{C} ; 58.01 \%$ yield; Anal. Calcd $\mathrm{C}_{19} \mathrm{H}_{16} \mathrm{~N}_{2} \mathrm{O}_{4}(336.0 \mathrm{~g})$ : $\mathrm{C}, 67.85 ; \mathrm{H}, 4.76$; N, 8.33; Found: C, 67.89; H, 4.72; N, 8.49; ${ }^{1} \mathrm{H}$ NMR $\delta$ $1.2\left(\mathrm{t}, 3 \mathrm{H}, \mathrm{CH}_{3}\right), 2.1\left(\mathrm{q}, 2 \mathrm{H}, \mathrm{CH}_{2}\right), 5.6(\mathrm{~s}, 1 \mathrm{H}, \mathrm{OH}), 7.0$ $7.8(\mathrm{~m}, 7 \mathrm{H}$, aromatic protons $), 8.0(\mathrm{~s}, 1 \mathrm{H},=\mathrm{CH}), 8.3(\mathrm{~d}$, $\left.1 \mathrm{H}, \mathrm{C}_{8}-\mathrm{H}\right), 9.5(\mathrm{~s}, 1 \mathrm{H}, \mathrm{NH})$; ms: m/z: $336\left(\mathrm{M}^{+}\right), 305$, 240, 200, 168, 148, 71, 69 and 57.

2.51 4-Ethyl-isocoumarin-3-carboxylic acid (3'-phenyl allylidene)-hydrazide (71): This compound was obtained as yellow crystals, mp: $172^{\circ} \mathrm{C} ; 50.00 \%$ yield; Anal. Calcd $\mathrm{C}_{21} \mathrm{H}_{18} \mathrm{~N}_{2} \mathrm{O}_{3}$ (346.0 g): C, 72.83; H, 5.20; N, 8.09; Found: C, 73.10; H, 5.41; N, 8.37; ${ }^{1} \mathrm{H}$ NMR $\delta$ $1.1\left(\mathrm{t}, 3 \mathrm{H}, \mathrm{CH}_{3}\right), 1.9\left(\mathrm{q}, 2 \mathrm{H}, \mathrm{CH}_{2}\right), 5.8\left(\mathrm{~s}, 1 \mathrm{H},=\mathrm{C}_{2^{\prime}} \mathrm{H}\right)$, $6.5\left(\mathrm{~s}, 1 \mathrm{H},=\mathrm{C}_{3^{\prime}} \mathrm{H}\right), 7.5\left(\mathrm{~s}, 1 \mathrm{H},=\mathrm{C}_{1^{\prime}} \mathrm{H}\right), 7.0-7.6(\mathrm{~m}, 8 \mathrm{H}$, aromatic protons), $8.0(\mathrm{~s}, 1 \mathrm{H}, \mathrm{NH}), 8.4\left(\mathrm{~d}, 1 \mathrm{H}, \mathrm{C}_{8}-\mathrm{H}\right)$; ms: m/z: $347\left(\mathrm{M}^{+}+1\right), 259,232,217,145,130,116$, $115,103,77$ and 76.

\subsection{Antimicrobial activity}

Antibacterial activity of newly synthesized compounds was tested in vitro in bacterial strains, Staphylococcus aureus and Escherichia coli using serial agar dilution (cup plate method). ${ }^{15}$

The two microorganisms were cultured in dishes containing agar medium, four cups $(8 \mathrm{~mm})$ were put onto the dishes and each test compound $(0.1 \mathrm{ml}$ of $2 \mathrm{mg} / \mathrm{ml}$ ) was added into the cups under aseptic condition. Then the dishes were incubated at $37^{\circ} \mathrm{C}$ for $24 \mathrm{~h}$. The zone of inhibition of the growth of the bacteria, which were produced by diffusion of the compounds from the cup into the surrounding medium, was measured to evaluate the antibacterial activity. Each experiment was repeated twice. DMF was used as a positive control for the experiments and the results were compared against standard drug ampicillin.

Antifungal activity was performed in vitro against fungal strains Thielaviopsis paradoxa, Phomopsis mangiferae, Fusarium pallidoroseum, Colletotrichum capsici using Potato Dextrose Agar Medium (Poisoned Food Technique). ${ }^{16}$

The standard fungal culture $T$. paradoxa, $P$. mangiferae, $F$. pallidoroseum and $C$. capsici were grown on PDA slants at room temperature.

Mycelial growth inhibition of $T$. paradoxa, $P$. mangiferae, F. pallidoroseum and $C$. capsici was evaluated by the poisoned food technique, where the inhibition in growth of the fungal strain was observed on PDA. The stock solutions (1000 ppm) were made from each of the test compounds. The required percent concentrations of the compounds $(\mathrm{mg} / \mathrm{ml})$ were obtained by mixing the appropriate amount of the stock solution with $20 \mathrm{ml}$ of molten PDA. The amended PDA was poured into petri dishes and allowed to set.

An inoculum of the fungus obtained from 7 days old axenic culture, grown as above, was placed at the centre of the amended agar medium. Each experiment was performed in triplicate. The diameter of the fungal colony was measured after 4 days and then 7 days at $26+1^{\circ} \mathrm{C}$ and the $\%$ inhibition was calculated using the following equation:

$\%$ inhibition $=\frac{\text { Growth area in reference }- \text { growth area in sample }}{\text { Growth area in reference }} \times 100$.

\subsection{Analgesic activity}

Analgesic activity of the compounds was determined by Tail flick method. ${ }^{17}$ Mice of either sex weighing between 20 and $25 \mathrm{~g}$ which shows positive response were selected and divided into different groups with four mice in each group. The first group served as control which received $2 \%$ gum acacia. Second group 
served as standard which received analgin at a dose of $50 \mathrm{mg} / \mathrm{kg}$ body weight orally. Rest of the groups received test compounds at a dose of $50 \mathrm{mg} / \mathrm{kg}$ body weight of mouse, orally.

The tail of the mouse was dipped (up to $5 \mathrm{~cm}$ ) in a water bath at $55 \pm 0.7^{\circ} \mathrm{C}$. The time taken to withdraw the tail clearly out of water was considered as the reaction time with the cut-off time being $60 \mathrm{~s}$. The first reading was taken immediately after administration of the standard drug and test compounds and afterwards at the intervals of $30 \mathrm{~min}$. The response time was recorded and the results are described in tabular form.

\section{Results and discussion}

The reaction sequence leading to formation of title compounds is outlined in schemes 1 and 2. 4-Alkylisocoumarin-3-carboxylic acids were used as starting material to introduce different heterocyclic moieties in it.

$o$-Acyl benzoic acids 1 on condensation with bromodiethyl malonate 2 in the presence of anhy. $\mathrm{K}_{2} \mathrm{CO}_{3}$ gives 4-alkyl-isocoumarin-3-carboxylic acids. First, a diethyl malonate intermediate of isocoumarin was obtained, which on hydrolysis with glacial acetic acid and conc. hydrochloric acid leads to the desired acids $\mathbf{3 a}-\mathbf{b}^{14}$ (scheme 1). Isocoumarin-3-carboxylic acid on treatment with hydrazine hydrate in ethyl alcohol gets converted to isocoumarin-3-carboxylic acid hydrazide 4a-b.

The frequencies obtained in IR spectra for acid hydrazide are 1694, 1652, 3269, 1588 for $Y$ lactone, $\mathrm{C}=\mathrm{O}$ and $\mathrm{N}-\mathrm{H}$ (amide) and $\mathrm{N}-\mathrm{H}$ (amine), respectively.

The signals obtained in the ${ }^{1} \mathrm{H}$ NMR spectrum of $\mathbf{4 a}$ are $\delta 2.2\left(\mathrm{~s}, 3 \mathrm{H}, \mathrm{CH}_{3}\right), 6.9\left(\mathrm{~d}, 2 \mathrm{H}, \mathrm{NH}_{2}\right), 7.9(\mathrm{t}, 1 \mathrm{H}$, $\mathrm{NH})$, 7.6-7.8 (m, 3H, aromatic protons), $8.4(\mathrm{~d}, 1 \mathrm{H}$, $\left.\mathrm{C}_{8}-\mathrm{H}\right)$ and mass spectra also supported the structure by showing $\mathrm{m} / \mathrm{z}$ at $218\left(\mathrm{M}^{+}\right)$which corresponds to the molecular formula $\mathrm{C}_{11} \mathrm{H}_{10} \mathrm{~N}_{2} \mathrm{O}_{3}$ (scheme 1).

Further, on synthetic scheme involve condensation of acid hydrazide $\mathbf{4 a} \mathbf{a}-\mathbf{b}$ with acetylacetone in $\mathrm{HCl}$ leading to cyclization following Pal Knor pathway resulted in 5a-b (scheme 1).

IR frequencies obtained in the spectra are 1709, 1644, 1586, 1489 and $1244 \mathrm{~cm}^{-1}$ for $Y$ lactone, $\mathrm{C}=\mathrm{O}, \mathrm{C}=\mathrm{C}, \mathrm{C}=\mathrm{N}$ and $\mathrm{C}-\mathrm{N}$, respectively, which helps in establishing the structure as well as cyclization formation.

5b Shows signals in ${ }^{1} \mathrm{H}$ NMR at $\delta 1.3\left(\mathrm{t}, 3 \mathrm{H}, \mathrm{CH}_{3}\right)$, 2.7 (q, 2H, $\left.\mathrm{CH}_{2}\right), 2.9$ (s, 6H, $\left.\mathrm{CH}_{3}\right), 6.2(\mathrm{~s}, 1 \mathrm{H},=\mathrm{CH})$, 7.5-7.8 (m, 3H, aromatic protons), $8.3\left(\mathrm{~d}, 1 \mathrm{H}, \mathrm{C}_{8}-\mathrm{H}\right)$ and in mass spectra $\mathrm{m} / \mathrm{z}$ at $295\left(\mathrm{M}^{+}-1\right)$, corresponds to the molecular formula $\mathrm{C}_{17} \mathrm{H}_{16} \mathrm{~N}_{2} \mathrm{O}_{3}$.

Schiff bases form an important class of organic compound with a wide variety of biological properties. Development of new chemotherapeutic Schiff base is now attracting the attention of medical chemist as well as organic chemists too. Many studies have been reported on the biological activity of Schiff base such as antimicrobial, antifungal, herbicidal, anticancer derived from various heterocycles. To the best of our knowledge, Schiff base with isocoumain moiety has not yet been synthesized and its analgesic activities not studied. Keeping in view these importances in different biological systems, condensation of isocoumarin moiety was done with different aldehydes to yield Schiff bases (scheme 2).

Isocoumarin-3-carboxylic acid hydrazide 4 when refluxed with different substituted benzaldehyde $\mathbf{6}$ in ethanol in the presence of conc. sulphuric acid leads to formation of Schiff bases $\mathbf{7 a - l}$. The build up of $\mathbf{7 a - l}$ is evident from their spectral data.

The frequencies obtained for Schiff bases in IR spectra are $1703,1623,1597,1296 \mathrm{~cm}^{-1}$ corresponding to $\gamma$ lactone, $\mathrm{C}=\mathrm{O}, \mathrm{C}=\mathrm{N}$ and $\mathrm{C}-\mathrm{N}$, respectively.

${ }^{1} \mathrm{H}$ NMR spectra of $\mathbf{7 c}$ and $\mathbf{7 h}$ show signals at $\delta 2.0$ (s, $\left.3 \mathrm{H}, \mathrm{CH}_{3}\right), 4.0\left(\mathrm{~s}, 3 \mathrm{H}, \mathrm{OCH}_{3}\right), 7.3(\mathrm{~s}, 1 \mathrm{H},-\mathrm{C}=\mathrm{H})$, $7.1-8.3(\mathrm{~m}, 7 \mathrm{H}$, aromatic protons $), 8.4\left(\mathrm{dd}, 1 \mathrm{H}, \mathrm{C}_{8}-\right.$ $\mathrm{H}), 9.3(\mathrm{~s}, 1 \mathrm{H}, \mathrm{NH})$ and $\delta 1.1\left(\mathrm{t}, 3 \mathrm{H}, \mathrm{CH}_{3}\right), 2.5(\mathrm{q}$, $\left.2 \mathrm{H}, \mathrm{CH}_{2}\right), 4.9(\mathrm{~s}, 1 \mathrm{H}, \mathrm{OH}), 7.3(\mathrm{~s}, 1 \mathrm{H},=\mathrm{CH}), 7.1-8.3$ (m, $7 \mathrm{H}$, aromatic protons), $8.45\left(\mathrm{~d}, 1 \mathrm{H}, \mathrm{C}_{8}-\mathrm{H}\right), 10.1$ (s, $1 \mathrm{H}, \mathrm{NH})$.

Mass spectra of 7c show $\mathrm{m} / \mathrm{z}$ at $336\left(\mathrm{M}^{+}\right)$and $\mathbf{7 h}$ at $\mathrm{m} / \mathrm{z} 336\left(\mathrm{M}^{+}\right)$, corresponding to molecular formulae $\mathrm{C}_{19} \mathrm{H}_{16} \mathrm{~N}_{2} \mathrm{O}_{4}$ and $\mathrm{C}_{19} \mathrm{H}_{16} \mathrm{~N}_{2} \mathrm{O}_{4}$, respectively.

\subsection{Biological activity}

The title compounds synthesized $\mathbf{4 a - b}, \mathbf{5 a}-\mathbf{b}$ and $\mathbf{7 a - 1}$ were tested in vitro for antibacterial activity against bacterial strains Staphylococcus aureus (Gram positive) and Escherichia coli (Gram negative). Zone of inhibition was measured to determine the activity and ampicillin was used as the standard drug and DMF as control (schemes 1 and 2).

The investigation of the antimicrobial screening of compounds $\mathbf{4 a}-\mathbf{b}, \mathbf{5 a}-\mathbf{b}$ and $\mathbf{7 a}-\mathbf{l}$ revealed that all the synthesized compounds showed moderate to good antibacterial activity against $E$. coli.

Isocoumarin-3-carboxylic acid hydrazide $\mathbf{4 a - b}$, has enhanced zone of inhibition than corresponding acid indicating better antibacterial activity than parent compound. 


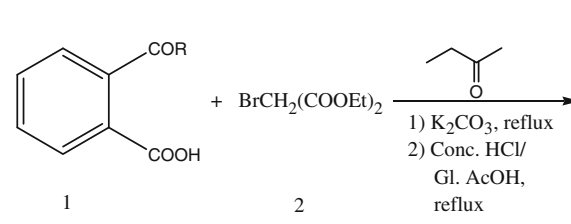

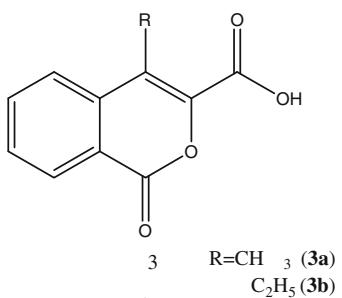<smiles>[2H]c1c(C(=O)n2nc(C)cc2C)oc(=O)c2ccccc12</smiles><smiles>[2H]c1c(C(=O)NCCCCCCC(C)C)oc(=O)c2ccccc12</smiles><smiles>[2H]c1c(C(=O)N2N=C(C)C([2H])(CC)C2(C)C)oc(=O)c2ccccc12</smiles>

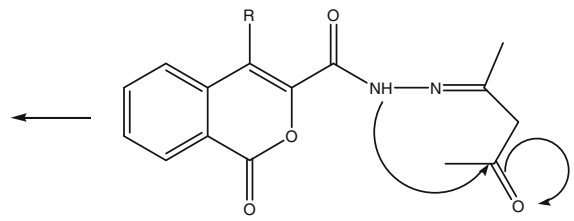
$\downarrow-\mathrm{H}_{2} \mathrm{O}$<smiles>[2H]c1c(C(=O)n2nc(C)cc2C)oc(=O)c2ccccc12</smiles>

Scheme 1. Mechanism of dimethyl pyrazole -1-carbonyl isocoumarin derivative.

Pyrazole derivative of isocoumarins seems to be more effective against $S$. aureus than $E$. coli.

On the other hand, Schiff bases of isocoumarins show exceptionally good results against $S$. aureus and better activity with $E$. coli, enhancing the importance of carbon-nitrogen double bond system. Conjugation between isocoumarins and phenyl ring were also found to enhance the activity.

The synthesized compounds were screened for the antifungal activity against fungal strains, Fusarium pallidoroseum, Colletotrichum capsici, belonging to the same family, using Potato Dextrose Agar Medium. ${ }^{16}$ 

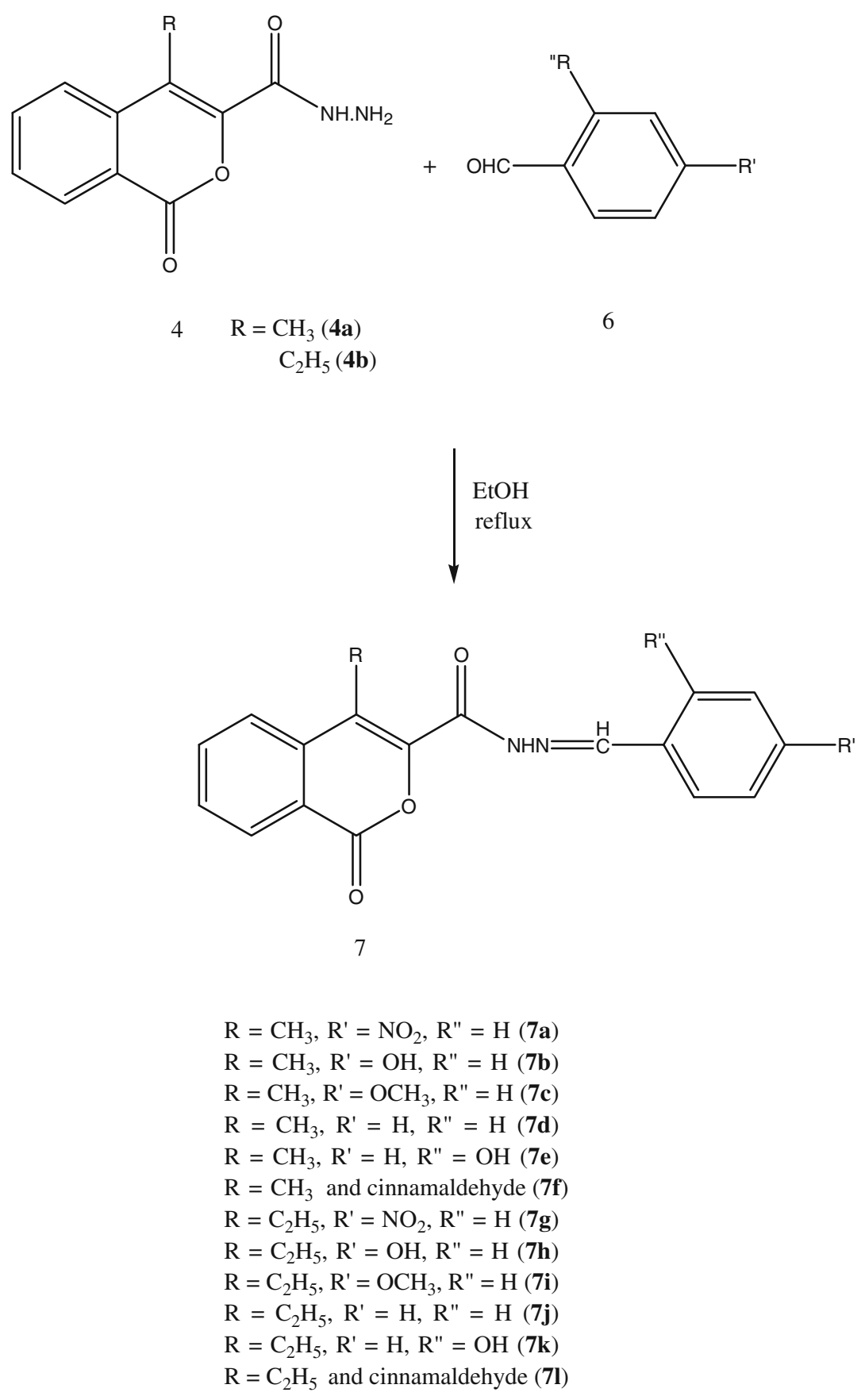

Scheme 2. Synthesis of Schiff base isocoumarin derivatives.

In antifungal activity, with increase in length of alkyl chain at 4th position of 4-alkyl-isocoumarin-3carboxylic acid, slight decrease in activity is observed. Acid hydrazide is found to show the same activity as the parent compound acid has. Schiff bases of isocoumarins furnish good quality results in opposition Chaetomium and $F$. pallidorosoeum strains. Here, length of alkyl chain has no effect on activity. The observed data on the antimicrobial activity of the compounds and control drugs is given in table 1 .
Analgesic activity of the compounds was determined by tail flick method ${ }^{17}$ on mice of either sex. $2 \%$ Gum acacia was used as the control, Analgin was the standard drug and their reaction time being 3.00 and $9.00 \mathrm{~s}$, respectively (table 2 ).

Isocoumarin-3-carboxylic acid hydrazide, Pyrazole and Schiff base derivatives give extremely good results, very similon to the reaction time of standard, enhancing the importance of $\mathrm{C}=\mathrm{N}$ bond in biological systems. Remarkably good result in $\mathbf{7 f}$ might be due to conjugation. 
Table 1. Antimicrobial activity.

\begin{tabular}{|c|c|c|c|c|}
\hline \multirow[b]{2}{*}{ Compound } & \multicolumn{2}{|c|}{ Zone of inhibition (mm) } & \multicolumn{2}{|c|}{$\%$ Growth of inhibition } \\
\hline & S. aureus & E. coli & F. pallidoroseum & Chaetonium \\
\hline $4 \mathbf{a}$ & 14 & 14 & 58.73 & 55.30 \\
\hline $4 b$ & 13 & 12 & - & - \\
\hline $5 \mathbf{a}$ & 11 & 15 & 44.76 & 49.11 \\
\hline $5 \mathbf{b}$ & 12 & 11 & 40.00 & 62.90 \\
\hline $7 c$ & 15 & 14 & 75.07 & 77.90 \\
\hline $7 \mathbf{h}$ & 11 & 12 & 65.02 & 70.00 \\
\hline $7 f$ & 17 & 17 & 81.00 & 78.80 \\
\hline Control (DMF) & 0 & 10 & - & - \\
\hline Ampicillin & 15 & 5 & - & - \\
\hline
\end{tabular}

Table 2. Analgesic activity.

\begin{tabular}{|c|c|c|c|c|c|}
\hline \multirow[b]{2}{*}{ Compound } & \multirow[b]{2}{*}{ Dose $(\mathrm{mg} / \mathrm{kg})$ body weight } & \multicolumn{4}{|c|}{$\begin{array}{l}\text { Average }( \pm \mathrm{SE}) \text { reaction time }(\mathrm{s}) \\
\text { Time after drug treatment }(\mathrm{min})\end{array}$} \\
\hline & & 0 & 30 & 60 & 90 \\
\hline Control & 50 & $3.01( \pm 0.358)$ & $3.20( \pm 0.288)$ & $3.10( \pm 0.358)$ & $3.02( \pm 0.00)$ \\
\hline Standard & 50 & $3.09( \pm 0.408)$ & $5.25( \pm 0.249)$ & $7.75( \pm 0.249)$ & $9.00( \pm 0.000)$ \\
\hline $4 a$ & 50 & $3.27( \pm 0.277)$ & $3.98( \pm 0.153)$ & $6.74( \pm .540)$ & $8.14( \pm 0.560)$ \\
\hline $5 \mathbf{a}$ & 50 & $3.03( \pm 0.008)$ & $5.49( \pm 0.049)$ & $7.02( \pm 0.195)$ & $8.63( \pm 0.403)$ \\
\hline $7 c$ & 50 & $3.00( \pm 0.408)$ & $5.32( \pm 0.408)$ & $5.59( \pm 0.549)$ & $7.31( \pm 0.408)$ \\
\hline $7 \mathbf{h}$ & 50 & $2.00( \pm 0.308)$ & $4.00( \pm 0.500)$ & $5.19( \pm 0.353)$ & $6.84( \pm 0.500)$ \\
\hline $7 f$ & 50 & $3.12( \pm 0.049)$ & $4.93( \pm 0.322)$ & $8.00( \pm 0.249)$ & $8.50( \pm 0.249)$ \\
\hline
\end{tabular}

\section{Conclusion}

This study reports the successful synthesis and antimicrobial, analgesic activities of new pyrazole and Schiff base analogues of isocoumarins. The divergence in the antibacterial activity of these compounds validates the significance of this study. The antibacterial activity study revealed that the most of the compounds tested showed moderate to good antibacterial activity. Schiff bases were more effective as antifungal agents. All the tested compounds showed good analgesic action. However, the effect of compounds on the host cell and their mode of action need to be studied.

\section{Acknowledgements}

The authors thank the Sun Pharmaceutical Industries Ltd. Baroda and Sophisticated Analytical Instrumentation Facility (SAIF), Punjab University for NMR spectra analysis. Thanks are also due to Prof. Anjana Desai, Mrs. S Aparna of Microbiology Department, the MS University of Baroda for antibacterial screening, as well as to Prof. Arun Arya, Head, Department of Botany, The MS University of Baroda, for antifungal screening, and to Mr. G Paramesh, Gulberga University for the help in analgesic activity study.

\section{References}

1. Ouyang G, Chen Z, Cai X J, Song B A, Bhadury P S, Yang S, Jin L H, Xue W, Hu, D Y and Zeng S 2008 Bioorg. Med. Chem. 169699

2. Bandaya M R, Mattoob R H and Rauf A 2010 J. Chem. Sci. 122177

3. Gokhan-Kelekc N, Yabanoglu S, Kupeli E, Salgın U, Ozgen O, Ucar G, Yesilada E, Kendi E, Yesilada A and Bilgin A A 2007 Bioorg. Med. Chem. 15 5775

4. Abdel-Hafez E S M N, El-Din G, Abuo-Rahma A A, Abdel-Aziz M, Radwan M F and Farag H H 2009 Bioorg. Med. Chem. 173829

5. Padmaja A, Payani T, Reddy G D and Padmavathi V 2009 Eur. J. Med. Chem. 444557

6. Chen H J, Liu Y, Wang L N, Shen Q, Li J and Nan F J 2010 Bioorg. Med. Chem. Lett. 202876

7. Yan R Z, Liu X Y, Xu W F, Pannecouque C, Witvrouw $\mathrm{M}$ and De Clercq E 2006 Arch. Pharm. Res. 29 957

8. Bandgar B P, Gawande S S, Bodade R G, Gawande N M and Khobragade C N 2009 Bioorg. Med. Chem. 17 8168

9. Kaymakcioglu B K, Rollas S, Korcegez E and Aricioglu F 2005 Eur. J. Pharm. Sci. 2697

10. Pal A, Ghosh R, Adarsh N N and Sarkar A 2010 Tetrahedron 665451

11. Che C M and Huang J S 2003 Coordination Chem. Rev. 24297 
12. Przybylski P, Huczynski A, Pyta K, Brzezinski B and Bartl F 2009 Curr. Org. Chem. 13124

13. Da Silva C M, Da Silva D L, Modolo L V, Alves R B, De Resende M A, Martins C V B and de Fátima A 2011 J. Adv. Res. 21

14. Chatterjea J N, Jha H C and Chattopadhyay A K 1972 Tetrahedron Lett. 133409
15. Indu M N, Hatha A A M, Abirosh C, Harsha U and Vivekanandan G 2006 Brazilian J. Microbiol. 37153

16. Nene Y L and Thapliyal P L 1979 Fungicide in plant disease control (New Delhi: Oxford and IBH Pub. Co.) 507

17. Kulkarni S K 1993 Handbook of experimental pharmacology (New Delhi: Vallabh Prakashan) 50 